\title{
New evidence in public health and general practice
}

\author{
Andrea Alberto Conti · Beatrice Dilaghi · \\ Pietro Amedeo Modesti · Carlo Nozzoli
}

Published online: 31 March 2009

(C) SIMI 2009

\section{Life expectancy improves as a consequence of the lowering in the exposure to fine-particulate air pollution}

Recent evidence indicates the role of air pollution as a risk factor for cardiovascular diseases. United States researchers assessed the modifications in the expectancy of life associated with differential modifications in fine particulate air pollution in the USA in the course of the 1980s and 1990s. Information regarding demographic features, socioeconomic status and life expectancy was collected for more than 200 county units in 51 metropolitan zones and matched with data regarding air pollution for the late 1970s and early 1980s and the late 1990s and early 2000s. The association between decreases in air pollution and modifications in the expectancy of life was computed by the use of regression models; adjustment for modifications in demographic characteristics, in socioeconomic parameters and in proxy markers for the frequency of smoking was performed. The authors report that a lowering of $10 \mu \mathrm{g} / \mathrm{m}^{3}$ in fine particulate matter results in association with an

\footnotetext{
A. A. Conti $(\bowtie) \cdot$ P. A. Modesti

Department of Critical Care Medicine and Surgery, University of Florence and Don Carlo Gnocchi Foundation, IRCCS Florence, Viale Morgagni 85, 50134 Florence, Italy e-mail: aa.conti@dac.unifi.it

B. Dilaghi

Department of Emergency Medicine, Azienda Ospedaliero-Universitaria Careggi,

Florence, Italy

C. Nozzoli

General Medicine Unit,

Azienda Ospedaliero-Universitaria Careggi,

Florence, Italy
}

estimated elevation in mean life expectancy of 0.61 year $(P=0.004)$. Air pollution lowering explained approximately $15 \%$ of the general augmentation of life expectancy in the zones explored by this study and the researchers concluded that life expectancy in the USA improved as a consequence of the lowering in the exposure to environmental fine-particulate air pollution.

\section{Reference}

Pope CA III, Ezzati M, Dockery DW (2009) Fine-particulate air pollution and life expectancy in the United States. N Engl J Med 360:376-386

In China too smoking is a powerful risk factor for mortality, in particular because of neoplastic, cardiovascular and respiratory diseases

In developed countries, smoking is a well-known risk factor for a number of diseases, including cardiovascular and neoplastic diseases, and its frequency is becoming higher in many developing areas of our planet. Chinese investigators designed and ran a large cohort study in a representative group of almost 170,000 Chinese people aged at least 40 years. Information on smoking habits and additional risk factors was collected by the researchers involved in the China National Hypertension Survey, both at baseline in 1991 and during the follow up in 1999 and 2000. The mortality attributable to smoking in 2005 was calculated, and a notable dose response association between the pack-years smoked and mortality attributable to every cause was recorded in males and in females after adjustment for many risk factors $(P<0.001$ for trend). 
Almost 700,000 $(673,000)$ deaths in China (with a 95\% confidence interval included between 564,700 and 781,400) appeared due to smoking, and in particular 134,800 deaths among females and 538,200 among males. Major determinants of mortality associated with smoking were neoplastic diseases (268,200 deaths), cardiovascular pathologies $(146,200)$ and respiratory diseases $(66,800)$. In China too, therefore, smoking is a powerful risk factor for death, requiring large education programs targeted toward the general population.

\section{Reference}

Gu D et al (2009) Mortality attributable to smoking in China. N Engl J Med 360:150-159

\section{Lower quantity and worse quality of sleep are associated with a higher probability of cold development in adult volunteers exposed to rhinoviruses}

A group of US researchers have recently studied the relationship between sleep and cold, assessing 153 adult volunteers, aged 21-55, who, in the course of two consecutive weeks, reported the total duration of their sleep, the percentage of bed time spent really sleeping (sleep efficiency) and their perception of their own sleep. After this 14-day period, the same individuals were administered nasal drops containing rhinoviruses and were strictly followed-up so as to observe the development, if present, of cold for 5 days after the exposure to the microbiological agents. A graded association of common cold with average sleep duration was documented, since individuals sleeping less than $7 \mathrm{~h}$ per night were almost 3 times $(2.94,95 \%$ confidence interval between 1.18 and 7.30) more likely to develop a cold than people sleeping at least $8 \mathrm{~h}$ per night. A graded association was observed also with reference to sleep efficiency, on account of the fact that individuals with an efficiency lower than $92 \%$ had a subsequent cold frequency more than 5 times $(5.50,95 \%$ confidence interval between 2.08 and 14.48) higher than subjects with a sleep efficiency of at least $98 \%$. Even, if the proportion of days in which the participants felt rested in the studied period was not associated with cold, the authors conclude that a decreased quantity and a lower quality of sleep are associated with a higher frequency of subsequent development of common cold in adult volunteers exposed to rhinoviruses.

\section{Reference}

Cohen S et al (2009) Sleep habits and susceptibility to the common cold. Arch Intern Med 169:62-67

\section{Hypertension, elderly age and female gender increase the response to dietary sodium interventions}

A group of US researchers investigated elements linked to the response of blood pressure to dietary interventions, in particular, regarding sodium and potassium, by performing a study including a week-long low-salt intervention (51.3 mmol sodium/day), a week-long high-salt intervention $(307.8 \mathrm{mmol}$ sodium/day), and a week-long high-salt and potassium-supplementation (60 $\mathrm{mmol}$ potassium/day) intervention. The participating subjects were more than 1,900 individuals from China, and their blood pressure was registered 9 times in the course of the initial 3 observation days and in the course of the last $72 \mathrm{~h}$ of every intervention stage. The reduction in blood pressure resulted greater in females than in males both for systolic pressure $(-8.1 \mathrm{mmHg}$, with $95 \%$ confidence interval between -8.6 and -7.6 , as compared to $-7.0 \mathrm{mmHg}$, with $95 \%$ confidence interval between -7.5 and -6.6 ) and for diastolic pressure $(-4.5 \mathrm{mmHg}$, with $95 \%$ confidence interval between -4.9 and -4.1 , as compared to $-3.4 \mathrm{mmHg}$, with $95 \%$ confidence interval between -3.8 and -3.0 ). In a similar way, the responses of blood pressure to highsodium interventions were significantly higher in females for systolic pressure $(6.4 \mathrm{mmHg}, 95 \%$ CI 5.9-6.8) than in males $(5.2 \mathrm{mmHg}, 95 \%$ CI $4.8-5.7)$ and for diastolic pressure $(3.1 \mathrm{mmHg}, 95 \% \mathrm{CI} 2.7-3.5$ as compared to $1.7 \mathrm{mmHg}, 95 \%$ CI $1.4-2.1)(P<0.001$ for every comparison). The authors of this study documented that the response of systolic blood pressure to sodium interventions increased with age, and that systolic and diastolic blood pressure responses to sodium interventions increased with initial blood pressure levels, as did blood pressure responses to potassium supplementation.

The presence of hypertension, elderly age and female gender heightens the sensitivity to dietary sodium interventions.

\section{Reference}

He $J$ et al (2009) Gender difference in blood pressure responses to dietary sodium intervention in the GenSalt study. J Hypertens 27:48-54 
In subjects with peripheral arterial disease, treadmill training improves quality of life, walking performance and flow-mediated dilation of the brachial artery

US researchers performed a randomized controlled trial in an academic health care centre to evaluate if the resistance training of lower extremity or the treadmill exercise could increase the functional performance of subjects affected by peripheral arterial disease. Between April 2004 and August 2008, 156 individuals with peripheral arterial disease were allocated in a random way to the resistance training of the lower extremity, to the treadmill exercise, or to the control group. The mean improvement in the flow-mediated dilation of the brachial artery in the treadmill group was $1.53 \%$ [95\% confidence interval (CI) $0.35-2.70 \% ; P=0.02$ ] if compared to the control group. The patients allocated to the treadmill exercise group had an increase, with regard to the 6' walk, of $35.9 \mathrm{~m}$ (95\% CI, $15.3-56.5 \mathrm{~m} ; P<0.001)$ in their walked distance, if compared to the subjects of the control group; the patients of the resistance training group showed an increase of $12.4 \mathrm{~m}$ (95\% CI, -8.42 to $33.3 \mathrm{~m}$; $P=0.24)$ in their walked distance, if compared to the patients in the control group. If compared to the control group, the treadmill group showed higher augmentations in the walking impairment distance score $(10.7 ; 95 \% \mathrm{CI}$, $1.56-19.9 ; P=0.02)$, in the maximal treadmill walking time (3.44 min; 95\% CI, 2.05-4.84 $\mathrm{min} ; P<0.001$ ) and in the SF-36 PF score $(7.5$; 95\% CI $0.00-15.0 ; P=0.02)$. Compared to the control group, the resistance training group showed higher increases in the walking impairment scores for distance $(6.92 ; 95 \%$ CI $1.07-12.8 ; P=0.02)$, in the maximal treadmill walking time $(1.90 \mathrm{~min}$; $95 \% \mathrm{CI}$ 0.49-3.31 min; $P=0.009$ ), in the SF-36 PF score (7.5; 95\% CI $0.0-15.0 ; P=0.04)$ and in stair climbing ability (10.4; 95\% CI 0.00-20.8; $P=0.03$ ).

The lower extremity resistance training was able to improve, in this study, the quality of life, the functional performance (measured by means of the treadmill walking) and the stair climbing ability, while treadmill training improved the treadmill walking performance, the 6' walk performance, the quality of life and the brachial artery flow-mediated dilation in patients affected by peripheral arterial disease with and without intermittent claudication.

\section{Reference}

McDermott MM et al (2009) Treadmill exercise and resistance training in patients with peripheral arterial disease with and without intermittent claudication: a randomized controlled trial. JAMA 301:165-174

The application of a surgical safety checklist is associated with a $47 \%$ lowering in mortality and a $36 \%$ lowering in the rate of complications of surgical non-cardiac adult individuals

Surgical checklists have been elaborated to improve health staff communication and patient outcomes. In this study, a 19 item surgical safety checklist was proposed by an international research group in the context of the World Health Organization's Safe Surgery Saves Lives program. In the course of a year included between October 2007 and September 2008, eight hospitals in eight cities (Toronto, Canada; New Delhi, India; Amman, Jordan; Auckland, New Zealand; Manila, Philippines; Ifakara, Tanzania; London, England; and Seattle, USA), which represented different patient populations and socioeconomic characteristics, were analyzed so as to collect clinical information on processes and outcomes of more than 3,700 consecutive surgical adult patients recruited among individuals undergoing non-cardiac surgical interventions. Following this data collection, clinical information regarding more than 3,900 consecutive patients undergoing surgery after the introduction of the surgical safety checklist was documented, so as to explore the major end points of mortality rate and of complication rate. The rate of intra-hospital complications was $11.0 \%$ in patients at baseline and $7.0 \%$ after the implementation of the surgical checklist $(P<0.001)$; the rate of mortality was $1.5 \%$ before the introduction of the checklist and $0.8 \%$ after its introduction $(P=0.003)$. The authors conclude that the safety surgical checklist is a safe and effective tool to reduce complications and death rates in surgical non-cardiac patients in different hospitals.

\section{Reference}

Haynes AB et al (2009) The Safe Surgery Saves Lives Study Group. A surgical safety checklist to reduce morbidity and mortality in a global population. N Engl J Med 360:491-499

Conflict of interest statement The authors declare that they have no conflict of interest related to the publication of this manuscript 